\title{
A New Dimension of Codons for Protein Synthesis
}

\author{
Jacob Varghese B*, Khai Bui, Naomi Divall, Alexander Egan, Ali Kermani, Adam Lodge and Daniela Vollmer \\ Institute for Cell and Molecular Bioscience, Newcastle University, United Kingdom
}

\begin{abstract}
Protein synthesis is a series of codon-anticodon interactions to buildup the polypeptide chains. The corresponding rule is that out of 64 codons in mRNA, 61 codons have recognizing ability of the 20 amino acids based on degeneracy except methionine and tryptophan which are coded by single codon. The remaining three are stop codons of mRNA sequence, which does not decode any amino acids in the protein synthesis mechanism. A fascinating study is how many amino acids are recognizing the high guanine-cytosine or adenine-uracil rich triplet codons. These data would be useful to find the copy numbers of transfer RNA that encode the amino acids having high guanine-cytosine triplet codons or adenine-uracil rich triplet codons in three domains of life (Bacteria, Archeae and eukaryotes). The four types of nucleotides compositions (guanine, cytosine, adenine and thymine or uracil) have distinctly playing a central role in amino acid composition of protein sequences.
\end{abstract}

Keywords: Codon; Anti-codon; Amino acids; tRNA

\section{Introduction}

Transfer Ribonucleic acids (tRNA) is a polymer of four different types of nucleotides such as guanine and adenine cytosine and uracil. Each nucleotide is made of an invariant phosphorylated sugar to which is attached one of the four standard nucleic acids [1]. tRNA has the clover leaf secondary structure with the acceptor, anticodon, D-arm and T-arm which are folded into the L-shaped tertiary structure. tRNA structure have nearly 79 modifications that have been identified and characterized in all three domains. The modified tRNA's function is to improve the fidelity and efficiency in protein synthesis by maintains the reading frame [2]. tRNA anticodon is unique on each tRNA because triplet anticodons (combination of $\mathrm{G}, \mathrm{C}, \mathrm{A}, \mathrm{U}$ ) buildup a polypeptide chain by adding 20 different amino acids (Table 1). Possibly, high GC ratios of tRNA anticodon synthesis the polypeptide chain have high percentage of proline, arginine, alanine and glycine in protein molecules. However, high AU ratio of tRNA anticodons synthesis the protein molecules which have been more number of phenylalanine, Leucine, Isoleucine, Methionine, Tyrosine, Asparagine and Lysine. For example, high GC content organism have distinct synonymous of codon usages and tRNA subsets that results as high frequency

\begin{tabular}{|c|c|c|c|c|c|c|c|c|c|}
\hline \multirow{2}{*}{$\begin{array}{c}1^{\text {st }} \text { position } \\
\text { (5' end) }\end{array}$} & \multicolumn{8}{|c|}{$2^{\text {nd }}$ position } & \multirow{3}{*}{$\begin{array}{c}3^{\text {rd }} \text { position } \\
\text { (3'end) } \\
U\end{array}$} \\
\hline & \multicolumn{2}{|c|}{ U } & \multicolumn{2}{|c|}{ C } & \multicolumn{2}{|c|}{ A } & \multicolumn{2}{|c|}{ G } & \\
\hline \multirow{4}{*}{ U } & UUU & PHE & UCU & SER & UAU & TYR & UGU & CYS & \\
\hline & UUC & PHE & UCC & SER & UAC & TYR & UGC & CYS & C \\
\hline & UUA & LEU & UCA & SER & UAA & STOP & UGA & STOP & A \\
\hline & UUG & LEU & UCG & SER & UAG & STOP & UGG & TRP & G \\
\hline \multirow{4}{*}{ C } & CUU & LEU & $\mathrm{CCU}$ & PRO & CAU & HIS & CGU & ARG & u \\
\hline & CUC & LEU & $\mathrm{CCC}$ & PRO & CAC & HIS & CGC & ARG & C \\
\hline & CUA & LEU & CCA & PRO & CAA & GLN & CGA & ARG & A \\
\hline & CUG & LEU & CCG & PRO & CAG & GLN & CGG & ARG & G \\
\hline \multirow{4}{*}{ A } & AUU & IIE & ACU & THR & AAU & ASN & AGU & SER & $U$ \\
\hline & $A \cup C$ & IIE & ACC & THR & AAC & ASN & AGC & SER & C \\
\hline & AUA & IIE & ACA & THR & AAA & LYS & AGA & ARG & A \\
\hline & AUG & MET & ACG & THR & AAG & LYS & AGG & ARG & G \\
\hline & GUU & VAL & GCU & ALA & GAU & ASP & GGU & GLY & $u$ \\
\hline & GUC & VAL & GCC & ALA & GAC & ASP & GGC & GLY & C \\
\hline & GUA & VAL & GCA & ALA & GAA & GLU & GGA & GLY & A \\
\hline & GUG & VAL & GCG & ALA & GAG & GLU & GGG & GLY & G \\
\hline
\end{tabular}

High GC nucleotides codon bases were colored in blue whereas $A U$ rich nucleotides codons were colored in red. According GC rich and AU rich triplet codons the amino acids were colored accordingly.

Table 1: Separation of tRNA codons based on high GC and AU rich nucleotides.

\begin{tabular}{|c|c|c|c|c|c|c|c|}
\hline S. No & A.A & 3nt GC & $\begin{array}{c}\text { 5' and middle } \\
\text { position } \mathrm{G} / \mathrm{C} \text {, } \\
3^{\prime} \text { is } \mathrm{A} / \mathrm{U}\end{array}$ & S. No & A.A & 3nt UA & $\begin{array}{c}5^{\prime} \text { and middle } \\
\text { position A/U, } \\
3^{\prime} \text { is G/C }\end{array}$ \\
\hline \multirow{2}{*}{1} & \multirow{2}{*}{ PRO } & $\mathrm{CCC}$ & $\mathrm{CCU}$ & 1 & PHE & UUU & UUC \\
\hline & & CCG & CCA & 2 & LEU & UUA & UUG \\
\hline \multirow{2}{*}{2} & \multirow{2}{*}{ ARG } & CGC & CGU & 3 & IIE & AUU AUA & $A \cup C$ \\
\hline & & CGG & CGA & 4 & MET & NIL & AUG \\
\hline \multirow{2}{*}{3} & \multirow{2}{*}{ ALA } & GCC & GCU & 5 & TYR & UAU & UAC \\
\hline & & GCG & GCA & 6 & TRP & UAA(STOP) & UAG \\
\hline \multirow[t]{2}{*}{4} & \multirow{2}{*}{ GLY } & GGC & GGU & 7 & ASN & AAU & AAC \\
\hline & & GGG & GGA & 8 & LYS & AAA & AAG \\
\hline
\end{tabular}

Four types of 8 codons were separated (Table 2) by high GC and AU rich nucleotides The first type 8 codons are GC triplet nucleotides means that 5' base, middle base and 3' bases (colored in blue). The $2^{\text {nd }}$ one is 5' and middle bases are CG the last 3 base is $U$ or $A$. Third type of 8 codons are $A U$ triplet nucleotides. The fourth type of 8 codons contains 5' and middle bases are AU, the last 3' base is $C$ or $G$.

Table 2: Segregation of GC rich, $A U$ rich codons and its amino acids.

number of positively charged (Arginine and Lysine), aromatic residues (Phenylalanine, Tryptophan, Tyrosine).

\section{Methodology}

In all 64 codons the four nucleotides ( $\mathrm{G}, \mathrm{C}, \mathrm{A}$ or $\mathrm{U}$ ) were equally distributed in the codons. Thus an interesting objective is how many amino acids code more numbers of high GC/AU bases codons. On this view, Proline, Arginine, Alanine and Glycine these four amino acids are unique features among 20 amino acids. In total 64 codons these four amino acids (P, R, A and G), each one have two numbers of triplet GC codons (Table 2). Whereas only one amino acid Isoleucine have two numbers of triplet AU codons other amino acids like Phenylalanine, Leucine, Tyrosine, Asparagine and Lysine have sole number of triplet AU codons.

Corresponding author: Jacob Varghese B, Institute for Cell and Molecular Bioscience, Newcastle University, Richardson Road, Newcastle upon Tyne, NE2 4AX, United Kingdom, E-mail: vbjacob6@gmail.com

Received October 24, 2012; Accepted December 24, 2012; Published December 26, 2012

Citation: Jacob Varghese B, Bui K, Divall N, Egan A, Kermani A, et al. (2012) A New Dimension of Codons for Protein Synthesis. J Metabolic Synd 1:117. doi:10.4172/2167-0943.1000117

Copyright: ( 2012 Jacob Varghese B, et al. This is an open-access article distributed under the terms of the Creative Commons Attribution License, which permits unrestricted use, distribution, and reproduction in any medium, provided the original author and source are credited. 


\begin{tabular}{|c|c|c|c|c|c|c|}
\hline \multirow{2}{*}{ S. No } & A.A & $\begin{array}{c}\mathbf{5}^{\prime} \text { have G/C } \\
\text { middle and } \\
\text { 3' have A/U }\end{array}$ & $\begin{array}{c}\text { Middle } \\
\text { Base A/U } \\
\text { ntd }\end{array}$ & A.A & $\begin{array}{c}\text { Middle } \\
\text { Base G/C } \\
\text { ntd }\end{array}$ & $\begin{array}{c}\mathbf{5} \text { ' have A/U, } \\
\text { middle and } \\
\text { 3' have G/C }\end{array}$ \\
\hline \multirow{2}{*}{1} & LEU & CUU & CUC & SER & UCU & UCC \\
\cline { 2 - 7 } & LEU & CUA & CUG & SER & UCA & UCG \\
\hline 2 & VAL & GUU & GUC & THR & ACU & ACC \\
\hline \multirow{2}{*}{3} & VAL & GUA & GUG & THR & ACA & ACG \\
\hline \multirow{2}{*}{3} & HIS & CAU & CAC & CYS & UGU & UGC \\
\hline 4 & GLN & CAA & CAG & STOP & UGA & UGG \\
\hline & ASP & GAU & GAC & SER & AGU & AGC \\
\hline
\end{tabular}

Another four types of 8 codons were separated on the basis of GC/AU base distribution on the respective amino acids. $L, V, H, Q, D, E, S, T, C, R$ these 10 amino acids shares the equal distribution of $G, C, A$ and $U$ nucleotides including two stop codons.

Table 3: Segregation of $A U$ and $G C$ rich triplet codons and its amino acids.

Among 64 codons, two forms of 32 codons are separated on bases of high GC or AU rich nucleotide distribution. The first forms of 32 codons are segregated into four types of 8 codons.

\section{Discussion}

In this method 32 codons are separated by four types of 8 codons on the basis of GC/AU nucleotides allocation. All four types of 8 codons has equal number of GC/AU units which decode the 10 different amino acids decoded Leu, Val, His, Gln, Asp, Glu, Ser, Thr, Cys, Arg including two stop codons (Table 3). Serine has six triplet codons (6 codons) which contain equal numbers of GC and AU nucleotides. Leu, Val, Thr these three amino acids have four triplet codons (12) with equal sharing of GC and AU nucleotides. His, Gln, Asp, Glu, Cys, Arg amino acids has two triplet codons (12) an equal sharing of GC and AU nucleotides. A stop codon has contains an equal share of two GC and AU triplet codons (2 codons).

\section{Conclusion}

The conclusion is G/C triplet codons encode just 4 amino acids but the $\mathrm{A} / \mathrm{U}$ codons encode 7 amino acids and one stop codon. Obviously these finding suggests that $A / U$ triplet codons encode more amino acids including stop codons. For example, E. coli has six codons of arginine that varies from 1.4 to 24.1 per 1000 codons that this codon bias is the difference among identical codon usage [3]. Glutamic acid, asparagines, isoleucine, phenylalanine and tyrosine are all encoded by $\mathrm{A} / \mathrm{T}$ rich codons and are used significantly less often in $M$. tuberculosis, whereas alanine, arginine, tryptophan and proline are much more frequent. It is conceivable that biased amino acid composition, which is a reflection of the skewed codon usage imposed by the high $\mathrm{G} / \mathrm{C}$ content of the genome [4].

\section{References}

1. Jacobs E, Mills JD, Janitz M (2012) The role of RNA structure in posttranscriptional regulation of gene expression. J Genet Genomics 39: 535543

2. Björk GR, Durand JM, Hagervall TG, Leipuviene R, Lundgren HK, et al. (1999) Transfer RNA modification: influence on translational frameshifting and metabolism. FEBS Lett 452: 47-51.

3. Gustilo EM, Vendeix FA, Agris PF (2008) tRNA's modifications bring order to gene expression. Curr Opin Microbiol 11: 134-140.

4. Tekaia F, Gordon SV, Garnier T, Brosch R, Barrell BG, et al. (1999) Analysis of the proteome of Mycobacterium tuberculosis in silico. Tuber Lung Dis 79 : 329-342. 